JOCHEN STROBEL

\title{
Eine digitale Edition der Korrespondenz August Wilhelm Schlegels
}

Im März bzw. Oktober 20 I 2 nahm das DFG-Projekt Digitalisierung und elektronische Edition der Korrespondenz August Wilhelm Schlegels in Dresden und in Marburg seine Arbeit auf. Unter den bedeutenden Autoren der Frühromantik ist der ältere Schlegel-Bruder wohl der in der Geschichte der Romantikforschung am meisten vernachlässigte. Die Defizite der Erschließung seines Nachlasses vor allem seit dem Zweiten Weltkrieg, also nach dem Erscheinen von Josef Körners Editionen, sind für das lange Zeit geringe Interesse der Forschung mitverantwortlich. Der durch die Kritische Friedrich-Schlegel-Ausgabe mit initiierte Boom ist August Wilhelm Schlegel nicht in vergleichbarer Weise zuteil geworden; zudem galten viele seiner Texte eher als spröde und professoral denn als originell.

Dabei hatte mit der Erwerbung des Nachlasses durch die Königliche Bibliothek Dresden I 873 und die dann einsetzenden philologischen Arbeiten Oskar Walzels (Dresden) und Josef Körners (Prag) die europäische Romantikforschung Ende des 19. und Anfang des 20. Jahrhunderts beeindruckende Impulse erhalten. Allerdings fehlten wichtige Briefkonvolute im Nachlass und die Forschung musste sich mit Teileditionen begnügen. Die fehlenden Nachlassteile konnten 1998 aus Schweizer Privatbesitz bei Christie's in London ersteigert werden; nach dem Auf- und Ausbau des Dresdner Digitalisierungszentrums sind inzwischen auch erste Werke aus dem Schlegel-Nachlass (Vorlesungen, Hamlet-Übersetzung) digitalisiert worden. ${ }^{1}$

Mit dem Ausbau eines Digitalisierungszentrums an der SLUB Dresden und der kooperativen Entwicklung eines Digitalisierungsworkflows auch für Nachlässe und Briefe sind die Voraussetzungen für eine Erschließung nach neuesten technischen und editorischen

1 http://digital.slub-dresden.de/ppn278337147 (I 2.05.20I2). 
146 Methoden nun gegeben. Das Projekt zielt auf die Verzeichnung, Digitalisierung und Edition aller in Dresden und andernorts liegenden Briefe von und an Schlegel. Auf bisherige Forschungsergebnisse wird aufgebaut, zugleich die Datenbasis erheblich erweitert. Das Referenzprojekt mit den Komponenten der Nachlasserschließung, der digitalen Präsentation der handschriftlichen und gedruckten Briefe (letztere als Images und im Volltext) einschließlich der Bereitstellung der dafür notwendigen Workflows sowie der Neuedition aller bisher ungedruckten Briefe gewährleistet die Anschlussfähigkeit für weitere Editionsprojekte.

Eine Auswertung des Dresdner Nachlasses sowie weiterer Bestände in der Dresdner Handschriftensammlung ergab eine Summe von etwa 3.200 Briefen, davon 585 im 1998 neu erworbenen Teilnachlass. Eine im Vorfeld weltweit bei 400 Archiven und Bibliotheken sowie über Suchwerkzeuge im Internet durchgeführte Recherche erbrachte noch einmal ca. I.000 Briefe von und an August Wilhelm Schlegel, die verstreut sind auf etwa 80 Institutionen. Mit Hunderten weiterer Briefe ist zu rechnen, insgesamt mit etwa 4.500 zugänglichen Briefhandschriften von und an A. W. Schlegel weltweit.

Da das vorläufige Briefrepertorium des Heidelberger Instituts für Textkritik, das I I 8 Publikationen bis 2005 auswertet, ${ }^{2} 2.350$ bereits edierte Briefe von und an August Wilhelm Schlegel auflistet, ist davon auszugehen, dass etwa 2.1 50 Briefe der Korrespondenz bis heute ungedruckt sind. Das Forschungspotential ist immens, berücksichtigt man, welche Namen in den 40 Bänden der Schlegel'schen Briefsammlung im Dresdner Nachlass enthalten sind. ${ }^{3}$

Abgesehen vor allem von Einzeleditionen der Korrespondenzen mit Friedrich Schlegel, Goethe, Wilhelm von Humboldt oder dem Bonner Schüler Christian Lassen, erschienen in den I $930 e r$ Jahren die drei bis heute nicht überholten Ausgaben, die A. W. Schlegels Briefe enthalten. Zwei davon legte der Prager Germanist Josef Körner vor, der sich über Jahrzehnte den Brüdern Schlegel, vor allem aber

2 http://www.textkritik.de/briefkasten/schlegel_a_w/schlegel_a_w_index.htm (12.05.2012).

3 Die Digitalisierung ist in Vorbereitung. Digitalisierte Teile des Nachlasses sind bereits zugänglich: http://digital.slub-dresden.de/ ( 12.05 .20 I 2). 
dem älteren der beiden, widmete. I 930 publizierte er zwei Bände mit 147 über 400 noch unveröffentlichten, reich kommentierten Briefen von und an Schlegel, ${ }^{4}$ in der seit 1936 erschienenen, insgesamt dreibändigen Sammlung Krisenjahre der Frühromantik finden sich 600 Briefe, die Körner 1929 in Schloss Coppet bei Genf, einst Madame de Staëls Wohnsitz und Schlegels langjähriger Aufenthaltsort, selbst entdeckt hatte.

Körner berichtet in seinen Bandeinleitungen von den jahrelangen Verzögerungen, die die Publikation hemmten. Den Zufall des Fundes von Coppet - es handelt sich um die 1998 von der SLUB erworbenen Bestände - und die daraus erwachsende Edition vermochte Körner umzudeuten zur Dokumentation eines zentralen, aus seiner Sicht positiv zu bewertenden Wendepunktes der deutschen Geistesgeschichte. Körners Editionen bezeichnen damit, trotz ihrer immensen Verdienste, zugleich einen wissenschaftlichen Horizont, an den heute nicht mehr angeknüpft werden kann:

Damit war die größte, die peinlichste Lücke im Quellenvorrat zur Geschichte der deutschen Romantik gefüllt und endlich jene Krisenzeit erhellt, in der sich mit der äußeren Auflösung der hochgeistigen Kreise von Jena und Berlin zugleich deren innere Abkehr vom maßlosen Subjektivismus und Ästhetizismus ihrer Anfänge vollzog und mit einer von vornherein anders ausgerichteten jüngeren Generation auch diese ältere zur Anerkennung von objektiven Bindungen des Bodens und der Geschichte, des Religiösen und Nationalen vorschritt, in ein unmittelbares Verhältnis zu den gegebenen Gemeinschaftsformen tretend. ${ }^{5}$

Ist Körners ideologischer Blickwinkel nicht unproblematisch, so ist die dritte große Sammlung von Schlegel-Briefen philologisch bedenklich: Mehr durch den Zufall der Überlieferung als durch editorische Professionalität bestimmt ist ausgerechnet Pauline de

4 Briefe von und an August Wilhelm Schlegel. Gesammelt und erläutert durch Josef Körner. Zwei Teile. Zürich/Leipzig/Wien 1930.

5 Josef Körner: [»Kurze Charakteristik «]. Zit. n.: »Vorbemerkung des Verlags «. In: Krisenjahre der Frühromantik. Briefe aus dem Schlegelkreis. Hg. v. Josef Körner. Dritter Band: Kommentar. Bern I 958, unpag.

Eine digitale Edition der Korrespondenz August Wilhelm Schlegels 
148 Panges Ausgabe der bedeutenden Briefe Friedrich und August Wilhelm Schlegels an Germaine de Staël und ihren Kreis. Die teils gekürzten Briefe sind in eine biographische Erzählung eingebettet; Briefe in französischer Sprache wurden in der erfolgreichen deutschsprachigen Ausgabe ins Deutsche rückübersetzt. ${ }^{6}$

Indessen sollen Josef Körners Verdienste nicht geschmälert werden. Er erweitert in seiner Edition von I 930 das Schlegel-Bild schon dadurch, dass er den Schwerpunkt nicht auf die Jenaer Zeit legt. Mit seiner Dreiteilung prägt der Band Schlegel drei Lebensphasen und auch drei Lebensrollen auf: »Kosmopolit der Kunst und Poesie « bis I 804 - dies wäre der bekannte Romantiker -, »Weltfahrer und Patriot « von I 804 bis I 8 I 8 - gleichsam Zwischenphase und Wanderjahre -, schließlich »Der deutsche Professor «. ${ }^{7}$ Der letztere Abschnitt, der die Bonner Jahrzehnte beleuchtet, ist verdienstvollerweise auch der umfangreichste. Körner würdigt Schlegel wohl erstmals vor allem als Gelehrten und erschließt in seinem Kommentar zahlreiche neue Quellen.

Hier möchte die jetzt begonnene Edition anschließen. Angeregt werden soll nicht nur die Erkundung von Beziehungsnetzen und Sozialfeldern des $>$ modernen $<$ Wissenschaftlers im I9. Jahrhundert, sondern auch die Erforschung einer durch Schlegel geprägten Interkulturalität. Müssen die in großem Umfang vorhandenen Daten zum Frühromantiker nur digital aufbereitet, zusammengeführt und vervollständigt werden, so mangelt es an Forschungsarbeiten zu Schlegel als Erzieher und international wirksamem Kommunikator in Coppet, als Politiker in Stockholm sowie als Professor in Bonn.

$\mathrm{Zu}$ entdecken ist vor allem der vielseitige Gelehrte August Wilhelm Schlegel, der neben Schelling, Ritter und Steffens wohl bedeutendste Fachwissenschaftler, den die Frühromantik hervorgebracht hat, einer der Mitbegründer der universitären Indologie in Deutschland. Schlegels Korrespondenz bezeugt einen wissenschaftlichen Paradigmenwechsel von einer universellen Sprach- und Literatur-

6 August Wilhelm Schlegel und Frau von Staël. Eine schicksalhafte Begegnung. Nach unveröffentlichten Briefen erzählt von Pauline de Pange. 5. Aufl. Hamburg 1940.

7 Briefe von und an August Wilhelm Schlegel (s. Anm. 4) (Zweiter Teil: Erläuterungen), S. I, S. I 85, S. 323. 
geschichte sowie Enzyklopädik zu einer Ausdifferenzierung und In- 149 stitutionalisierung philologischer Fachdisziplinen. Wissenschaftliche Vernetzung innerhalb der und zwischen den Disziplinen wie auch die Clusterbildung in Regionen oder Ländern artikulieren sich in der ersten Hälfte des I9. Jahrhunderts immer noch im Brief. Rekonstruieren lässt sich - mit dem virtuellen Brief-Zentrum Schlegel - ein europa-, wenn nicht weltweit agierendes Gelehrtennetzwerk, das Fach-, Institutionen- und Ländergrenzen teils erst (mit)generiert, teils auch schon wieder überschreitet und sprengt - und ein Forschungsgebiet, » wo alles noch im Werden begriffen ist «, wo »Entdeckungen täglich zu erwarten sind «, die, wie Schlegel in seiner Indischen Bibliothek einräumt, auch die »Theilnahme gebildeter Leser « erheischen, » welche aller wahren Erweiterungen des geistigen Besitzes sich lebhaft erfreuen. $\ll^{8}$

Die literatur- bzw. kulturwissenschaftliche Briefforschung hat sich zwar mit den vielfältigen Zusammenhängen zwischen Brief und Literatur befasst, sie hat jedoch für das I 9. Jahrhundert erst in Ansätzen den Brief als wissenschaftsgeschichtliche Quelle zu entdecken verstanden. ${ }^{9}$

Der wissenschaftsgeschichtliche Diskurs stellt sich seit etwa einem Jahrzehnt verstärkt der Frage nach $\gg$ den Darstellungsprinzipien [der] Analysen und Objekte, ihrer medialen Repräsentation und Reflexion « ${ }^{10}$ Joseph Vogl hatte mit einer »Poetologie des Wissens « nach dem »Auftauchen neuer Wissensobjekte und Erkenntnisbereiche zugleich als Form ihrer Inszenierung « gefragt. ${ }^{11}$ Welchen Stellenwert, so ist im Rahmen des geplanten Projekts zu fragen, kann

8 August Wilhelm Schlegel: »Vorrede «. In: ders.: Indische Bibliothek. Erster Band. Bonn I 820, S. IX-XV, hier: S. IX, S. XIII.

9 Vgl. Ulrich Johannes Schneider (Hg.): Kulturen des Wissens im I 8. Jahrbundert. Berlin/New York 2008.

10 Robert Matthias Erdbeer: »Deskriptionspoetik. Humboldts Kosmos, die verfahrensanalytische Methode und der wissenschaftsgeschichtliche Diskurs «. In: Bernhard J. Dotzler/Sigrid Weigel (Hg.): »fülle der combination «. Literaturforschung und Wissenschaftsgeschichte. München 2005, S. 239-266, hier: S. 239; vgl. zum $>$ cultural turn< in der Wissenschaftshistoriographie: Alf Lüdtke/Reiner Prass (Hg.): Gelehrtenleben. Wissenschaftspraxis in der Neuzeit. Köln u. a. 2008.

11 Joseph Vogl: »Einleitung «. In: ders. (Hg.): Poetologien des Wissens. Um I8oo. München I999, S. 7-16, hier: S. I3.

Eine digitale Edition der Korrespondenz August Wilhelm Schlegels 
150 der Brief, eine längst schon randständige und auch ästhetisch nicht mehr innovativ zu nennende Textsorte, im Wissenschaftsdiskurs nach i 800 noch einnehmen? Wie wird (literaturwissenschaftliches, sprachwissenschaftliches, orientalistisches ...) Wissen im Medium Brief artikuliert und inszeniert? Eine semantische Tiefenerschließung der Briefe, also eine differenzierte Sachverschlagwortung, die an die Stelle des ausführlichen Stellenkommentars tritt, soll die Benutzbarkeit für die Vertreter unterschiedlicher Fachdisziplinen gewährleisten.

Im Unterschied zu den bisherigen Romantiker-Briefeditionen wird erstmals ein größeres Briefkorpus ausschließlich als digitale Edition für open access aufbereitet. Entscheidend sind dabei die Erfordernisse einer internationalen scientific community, die via Internet mit den Projektergebnissen arbeiten wird. Aufgrund des Materialumfangs ist es weder denkbar noch für einen weiteren, durch das Projekt angeregten Forschungsprozess notwendig, dass in der Produktion sehr zeitaufwändige, bis in die Entstehungsvarianten vollständige und endgültige Editionen in Buchform vorgelegt werden. Ist das Instrument einer kritischen Edition für künstlerisch und wissenschaftlich diffizile Texte angemessen, deren komplexe Textentstehungs- und -überlieferungsprozesse mit ihren umfänglichen Umsemantisierungen zeichengenau abzubilden sind, so genügt für die Erschließung von August Wilhelm Schlegels Korrespondenz im Rahmen wissenschaftlicher Kommunikation ein die Quellen so gut wie möglich nachzeichnendes und erschließendes Verfahren. Die dem jeweiligen Nutzerinteresse anzupassende Variabilität in der Erschließungstiefe der Texte erlaubt es zugleich, die in sich heterogenen, teils schon älteren, aber vielfach philologisch zuverlässigen Printeditionen zusammen mit den Neutranskriptionen einzubinden. Erschließungskriterien sind, zusätzlich zur Transkription (die Entstehungsvarianten in Auswahl sichtbar machen soll): die Verschlagwortung von Namen, Werktiteln, Orten, Periodika und Sachen sowie biobibliographische Kurztexte zu allen Korrespondenten.

Das Projekt möchte maßgeblich am gegenwärtig zu beobachtenden ${ }^{12}$ Normierungsprozess für digitale (Brief-)Editionen mit-

12 Vgl. z. B. den Workshop Digitale Briefedition am 21./22.10.2010 in Berlin: http://hsozkult.geschichte.hu-berlin.de/termine/id= I 4539 (I 2.05.20 I 2); die 
wirken: Die Definition technischer Parameter sowie die Standards 151 der bibliothekarischen Formalerschließung (z. B. die Normierung von Metadaten) und fachwissenschaftlichen inhaltlichen Erschließung werden im Dialog mit der Fachcommunity vorangetrieben. Dies geschieht glücklicherweise zum Zeitpunkt einer neuen Hochkonjunktur philologisch ausgerichteter Schlegel-Forschung. Genannt seien die parallel zum hier vorgestellten Projekt fortgesetzte Kritische Ausgabe der Vorlesungen A. W. Schlegels (Prof. Dr. Georg Braungart; Dr. Stefan Knödler) und das DFG-Projekt Abschluss der Kritischen Friedrich-Schlegel-Ausgabe (Prof. Dr. Ulrich Breuer).

Die Arbeitsgruppe umfasst eine Bibliothekarin an der Sächsischen Landesbibliothek - Staats- und Universitätsbibliothek Dresden (SLUB; Projektleiter: Prof. Dr. Thomas Bürger), einen Informatiker am »Kompetenzzentrum für elektronische Erschließungs- und Publikationsverfahren in den Geisteswissenschaften « der Universität Trier sowie zwei Literaturwissenschaftlerinnen an der Philipps-Universität Marburg (Projektleiter: PD Dr. Jochen Strobel).

Informationen zum Projekt sind unter http://www.august-wilhelm-schlegel.de zu finden. Sowohl die Digitalisate des Dresdner Nachlasses als auch die Image-Digitalisate der bisherigen Drucke sind auf der Homepage der SLUB Dresden recherchierbar: http:// www.slub-dresden.de/sammlungen/. Fragen, Anregungen, nicht zuletzt aber Informationen über mutmaßlich noch unbekannte Briefe von und an August Wilhelm Schlegel werden erbeten an den Marburger Projektleiter (jochen.strobel@uni-marburg.de).

\footnotetext{
Weimarer Tagung Briefedition im digitalen Zeitalter am 5.-7.10.20 I I: http://hnet.msu.edu/cgi-bin/logbrowse.pl?trx $=v x \&$ list $=H$-Germanistik\& month= I I I 2 \&week=a\&msg=MOigkquqYEHUNfsRw93 AQw\&user $=\& p w=$ ( 2 2.05.20 I 2); die Brief-Sektion der Berner Tagung InterNationalität und InterDisziplinarität der Editionswissenschaft am I 5.- I 8.02.20 I 2: http://www.parzival.unibe.ch/Bern20 I2/index.html (I2.05. 2012) - sowie, mit einem Schwerpunkt auf Briefeditionen, die am I 8./ I 9.05.20 I 2 in Basel stattgefundene Tagung Online Editions. Problems and new Perspectives: http://www.ub.unibas.ch/ wiki/bernoulli/PIEGHEVOLE- I 8-I 9_maggio_ghelardi.pdf( ( 2 2.05.20 I 2).
}

Eine digitale Edition der Korrespondenz August Wilhelm Schlegels 